\title{
In situ magnetic measurements under neutron radiation in Fe metal and low carbon steel
}

\author{
S. Takahashi, ${ }^{a)}$ H. Kikuchi, and K. Ara \\ Nondestructive Evaluation and Science Research Center, Faculty of Engincering, Iwate University, Morioka \\ 020-8551, Japan \\ N. Ebine \\ Deparment of Reactor Safety Research, Japan Atomic Energy Agency, Tokai, Ibaraki 319-1195, Japan \\ Y. Kamada and S. Kobayashi \\ Nondestructive Evaluation and Science Research Center; Faculty of Engineering, Iwate University, Morioka \\ 020-855l, Japan \\ M. Suzuki \\ Department of Reactor Safety Research, Japan Atomic Energy Agency, Tokai, Ibaraki 319-1195. Japan
}

(Received 2 March 2006; accepted 8 May 2006; published online 20 July 2006)

\begin{abstract}
Magnetic minor hysteresis loops of low carbon steel and Fe metal have been measured during neutron radiation at $563 \mathrm{~K}$ in a $50 \mathrm{MW}$ nuclear reactor. For investigation of nucleation mechanism of copper precipitates and dislocation loops during neutron radiation. special attention was paid to minor-loop coefficients, which are deduced from simple relations between minor-loop parameters and are very sensitive to latlice defects such as dislocations, copper precipitates, and grain boundaries. We found that with increasing neutron fluence, the minor-loop coefficients of low carbon steel sharply increase and show a maximum at the fluence of $1 \times 10^{19} \mathrm{~cm}^{-2}$, followed by a slow decrease. The appearance of the maximum suggests the presence of two mechanisms of internal stress; while copper precipitates and dislocation loops in the matrix make the internal stress increase, those grown in the vicinity of dislocations compensate the internal stress of dislocations so as to minimize the elastic energy. On the other hand, the minor-loop coefficients show a monotonic increase with neutron fluence in Fe metal where dislocation loops play an important role for the brittleness. (c) 2006 American Institute of Physics. [DOI: 10.1063/1.2214214]
\end{abstract}

\section{INTRODUCTION}

The change of mechanical properties due to neutron irradiation has been studied for reactor pressure vessel materials and their model alloys. ${ }^{1}$ The neutron radiation induces several kinds of lattice defects such as vacancies, interstitial atoms, voids, dislocation loops, and copper precipitates. Some of the lattice defects make the ductility of materials low and make the brittleness increase. The structure of copper precipitates has been studied using an atom-probe field ion microscope $e^{2}$ and positron annihilation ${ }^{3}$ because copper precipitation is the primary cause of brittleness in nuclear reactor pressure vessels (NRPVs). It was revealed that copper precipitates with the size of $2-3 \mathrm{~nm}$, which are nucleated and grown by the irradiation, make materials brittle. ${ }^{4.5}$ However, the nucleation mechanism which takes into effect of dislocations has not been studied in detail, although the NRPV has high dislocation density $\rho$ even in the initial state. Dislocations would exert a great inlluence on the nucleation of lattice defects because there exists the elastic interaction between copper precipitates and dislocations. Our interest is in the nucleation mechanism of copper precipitates during neutron radiation. especially the interaction of radiation defects with dislocations in A533B steel. A533B steel is low

¿'Electronic mail: seiki.t@iwate-u.ac.jp carbon steel $(0.19$ wt $\%$ C, 0.19 wt $\%$ Si, $1.47 \mathrm{wt} \% \mathrm{Mn}$, 0.16 wt $\% \mathrm{Cu}, 0.64$ wt $\%$ Ni. 0.51 wt $\%$ Mo, 0.14 wt $\% \mathrm{Cr}$, balance $\mathrm{Fe}$ ) and is used in the NRPV.

The lattice defects can be observed directly or indirectly after neutron irradiation; the direct observation is due to a transmission electron microscope (TEM), while the indirect one is due to the magnetic method, ${ }^{6}$ the atom-probe field ion microscope, ${ }^{2}$ positron annihilation, ${ }^{3}$ small angle neutron scattering, ${ }^{7}$ electric resistance, ${ }^{1}$ etc. The information on radiation defects obtained by these currently employed methods is limited because current methods are used to measure physical properties only before and after the irradiation. The magnetic method has several advantages in getting information on lattice defects and can be technically applied for in situ measurements during neutron radiation in the nuclear reactor. The in situ observation of lattice defects by magnetic method gives us detailed information about irradiation damages against the neutron fluence. The main purpose of the present study is to perform magnetic measurements under neutron irradiation in the nuclear reactor.

The relationship between magnetism and dislocations in ferromagnetic metals was investigated by the Stuttgart group of Max-Planck Institute. ${ }^{8}$ The strain field around dislocations exerts an influence on magnetization hysteresis loops through the magneloelastic coupling in ferromagnetic metals such as $\mathrm{Ni}, \mathrm{Fe}$, and $\mathrm{Co}$ metals. The structure-sensitive properties change with the density and distribution of disloca- 
tions. The relationship of magnetic properties with other lattice defects such as vacancies, interstitial atoms, has not been studied in detail so far because these lattice defects bring about only a small change to the hysteresis loops; the size of these lattice defects is too small compared with the width of the magnetic domain wall to disturb the domain wall motion significantly.

Recently, an analysis method of minor hysteresis loops has been found in Fe single crystals, Fe polycrystals, and low carbon steel. ${ }^{9-12}$ Minor-loop coefficients deduced from simple relations between parameters of minor loops are independent of the magnetic field amplitude and are much more sensitive to dislocations and grain boundaries than the traditional magnetic properties of the major loop. In addition, one can obtain the minor-loop coefficients with much lower magnetic field compared with that of the major loop. For instance, $1 \mathrm{kA} / \mathrm{m}$ is sufficient for minor-loop analysis of A533B steel, though we need more than $10 \mathrm{kA} / \mathrm{m}$ for the major loop. ${ }^{12}$ Such low field measurements are very useful for present in situ magnetic measurements because the maximum applied field is limited to $2 \mathrm{kA} / \mathrm{m}$ owing to the limited space of an irradiation capsule for ring core samples.

The other purpose of the present study is to make a database of the relation between minor-loop coefficients and radiation defects. Currently, the integrity of pressure vessels is evaluated by Charpy impact test, which is a destructive testing. The lack of Charpy impact test samples preinstalled in the reactor is an urgent issue. On the other hand, the integrity can be evaluated nondestructively by the magnetic method. The database is necessary for the practical realization of the magnetic method for nondestructive evaluation of NRPV.

Copper precipitates and dislocation loops are main lat tice defects that cause the brittleness of NRPV during neutron radiation. In irradiated A533B steel, there coexist copper precipitates and dislocation loops, while only dislocation loops are induced by the radiation in Fe metal. In the present investigation, we studied radiation effects in A533B steel and Fe metal, and tried to separate the effects of these two lattice defects, in order to elucidale the relation between copper precipitates and minor-loop properties. The in situ measurements were performed at the same temperature as $563 \mathrm{~K}$ of the commercial reactor operation.

\section{ANALYSIS METHOD OF MINOR HYSTERESIS LOOPS}

To analyze minor hysteresis loops, we introduce minorloop properties as shown in Fig. 1; minor-loop magnetization $M_{a,}$, minor-loop coercive lorce $H_{c}^{*}$, minor-loop remanence $M_{R}$, minor-loop hysteresis loss $W_{F}^{*}$, minor-loop remanence work $W_{R}^{*}$, and ininor-loop susceptibilities $\chi_{H}{ }^{*}, \chi_{R}{ }^{*}$. and $\chi_{a}^{*}$. These parameters are used to represent characteristics of a set of minor hysteresis loops. They correspond to saturation magnetization $M_{s}$, coercive force $H_{c}$. remanence $M_{R}$, hysteresis loss $W_{F}$, remanence work $W_{R}$, and susceptibilities, in the major loop, respectively. $W_{R}$ "is the area enclosed by a minor loop in the second quadrant. We can obtain information about the potential energy of domain wall displacement by analyzing these minor-loop properties.

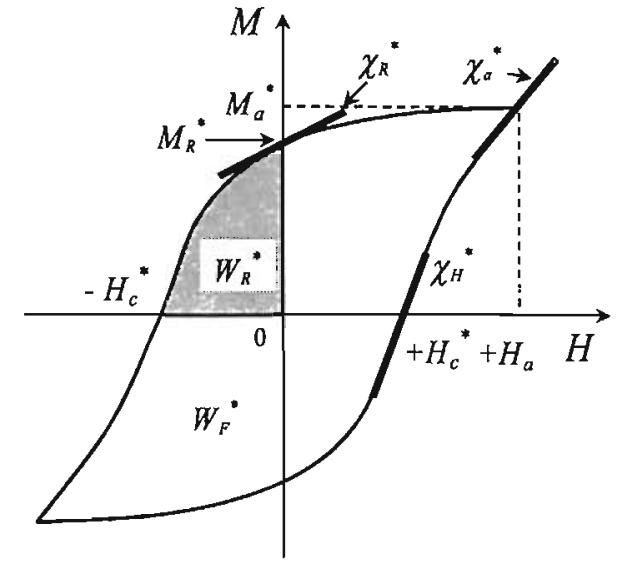

FIG. 1. The magnetic properties of a minor hysteresis loop; magnetic field amplitude $H_{a}$. minor-loop coercive force $H_{c}{ }^{*}$. minor-loop magnetization $M_{a}{ }^{*}$ at $H_{a}$, minor-loop hysteresis loss $W_{F}{ }^{*}$. minor-loop remanence $M_{R}{ }^{*}$, minorloop remanence work $W_{R}^{*}$, and three minor-loop susceptibilities $\chi_{H}{ }^{*}, X_{R}{ }^{*}$, and $X_{a}{ }^{*}$ at $H=H_{c}^{*}, 0$, and $H_{a}$, respectively.

We found six relations between the minor-loop properties in a set of minor loops. which are independent of the magnetic field amplitude $H_{a}$ as well as magnetic field. ${ }^{11.12}$ In the second stage where Bloch walls mainly contribute to the magnetization, $W_{F}^{*}$ and $W_{R}^{*}$ have a simple relation with $M_{u}^{*}$ and $M_{R}^{*}$, respectively, as follows:

$$
W_{F}^{* *}=W_{F}^{0}\left(\frac{M_{u}^{*}}{M_{s}}\right)^{n_{F}}
$$

and

$$
W_{R}^{*}=W_{R}^{0}\left(\frac{M_{R}^{*}}{M_{R}}\right)^{n_{R}} .
$$

Here, $W_{F}{ }^{\prime}$ and $W_{R}{ }^{\prime \prime}$ are coefficients representing the magnitude and the trough depth of Bloch wall potential, respectively, and are sensitive to lattice defects. $n_{F}$ and $n_{R}$ are constants independent of lattice defects as well as the kind of materials: $n_{F} \sim n_{R} \sim 3 / 2$. Note that the power-law relation with $n_{F}=1.6$ between $W_{F}$ and $M_{a}$ was discovered by Steinmetz about one century ago and is well-known as the Steinmetz law. ${ }^{13}$

$H_{c}^{*}$ corresponds to the height of the crest of potential and $M_{R}^{* *}$ represents the position of the trough in the vicinity of the crest. It was revealed that $H_{c}$ i has a simple relation with $M_{R}^{*}$ in the second stage, given by

$$
H_{C}^{*}=H_{C}{ }^{0}\left(\frac{M_{R}}{M_{R}}\right)^{{ }^{\prime C}} \text {. }
$$

where $H_{c}$ " is a coefficient representing the height of potential and $h_{c}$. is a constant which slightly depends on the kind of materials: $n_{c} \sim 0.45$.

In the initial stage of the magnetization process, three minor-loop susceptibilities $\chi_{H}{ }^{*}, \chi_{R}$. and $\chi_{A}$ are a function of $H_{r}^{*}, M_{R}$. and $M_{a}^{*}$, respectively. and are given by

$$
R_{C}^{*}=\frac{1}{\chi_{H}^{*}}=R_{C}^{0} \exp \left(-b \frac{H_{C}^{*}}{H_{C}}\right),
$$


(a)

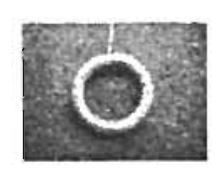

(b)

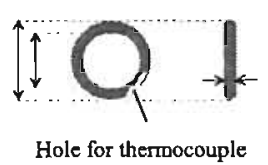

(c)

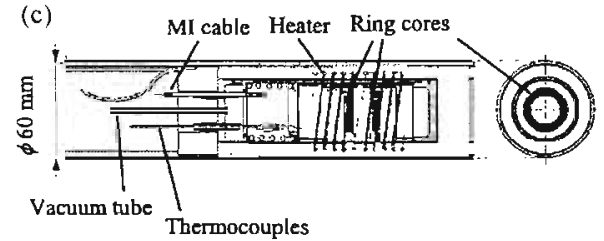

FIG. 2. (a) Pholograph and (b) size of a ring core sample. (c) Part of irradiation capsule equipped with ring cores.

$$
\chi_{R}^{*}=\chi_{T}^{0}\left(\frac{M_{R}^{*}}{M_{R}}\right)^{n_{T}}
$$

and

$$
\chi_{a}^{*}=\chi_{s}^{0}\left(\frac{M_{a}^{*}}{M_{s}}\right)^{n_{s}} .
$$

Here, $R_{c}{ }^{\circ}, \chi_{T}{ }^{\circ}$, and $\chi_{s}{ }^{\circ}$ are coefficients which represent the resistance at the crest, the mobility at the Irough. and the averaged mobility of Bloch wall, respectively. $b, n_{T}$, and $n_{s}$ are constants which depend on the kind of materials, and have a value of $2-6,0.1-0.5$, and $0.5-1.0$, respectively.

All minor-loop coefficients are sensitive to lattice defects compared with the structure-sensitive propesties of the major loop and have a proportional relation with coercive force. ${ }^{12}$ In the present study, the relations of Eqs. (1)-(6) which were obtained in plastically deformed Fe metal and A533B steel were adapted to the investigation of the effect of neutron radiation on minor-loop properties in Fe metal and $\mathrm{A} 533 \mathrm{~B}$ steel.

\section{EXPERIMENTAL PROCEDURE}

Sheet samples of Fe polycrystals with purity of $99.99 \%$ and $\mathrm{A} 533 \mathrm{~B}$ steel were prepared by cold rolling. During the manufacturing process, the steel was austenitized by aging at $1153 \mathrm{~K}$ for $\mathrm{l} \mathrm{h}$ and was tempered at $943 \mathrm{~K}$ for $80 \mathrm{~min}$ for the formation of bainite structure. The samples were shaped into a ring core with electric discharge machine for magnetic measurements as shown in Figs. 2(a) and 2(b).

Magnetic measurements of ring cores under neutron radiation were performed in Japan Material Testing Reactor at Japan Atomic Energy Agency. For in situ magnetic measurements, the ring cores with an exciting coil, pickup coil, and thermocouple, were installed into an irradiation capsule as shown in Fig. 2(c). Mineral insulated (MI) cables which have high durability against neutron irradiation were used for the coils. The MI cable with a diameter of $0.5 \mathrm{~mm}$ comprises a copper wire for the exciting coil, a constantan wire for the pickup coil, $\mathrm{MgO}$ insulator, and a stainless steel sheath. This wire was also used for thermocouples because of the high durability against neutron irradiation. This MI cable was wound on the ring core with 100 turns, which yields a maximum applied field of $2 \mathrm{kA} / \mathrm{m}$. The MI cables extended from

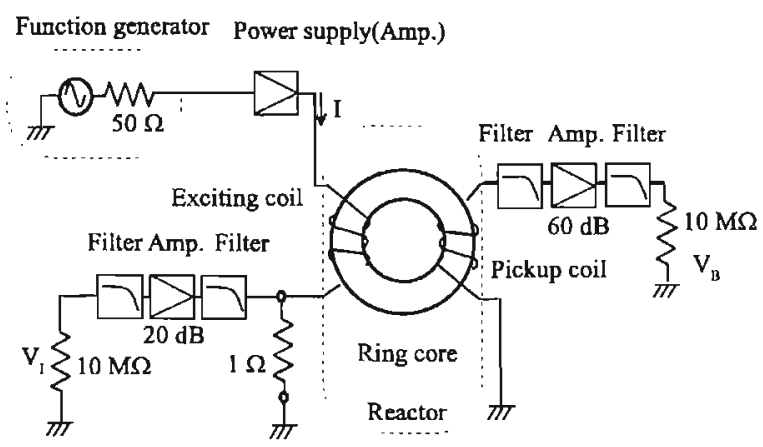

FIG. 3. Schematic diagram of electric circuit for minor-loop measurements.

the capsule were connected to an electronic circuit installed outside the reactor.

Figure 3 shows a schematic diagram of the electronic circuit for minor-loop measurements. A triangular voltage from a function generator was applied to a bipolar power supply. The bipolar power supply converts voltage to current and amplifies the current. The amplitied current was applied to the exciting coil to magnetize the ring cores with a rate of $400 \mathrm{~A} \mathrm{~m}^{-1} \mathrm{~s}^{-1}$. The magnetic field inside the ring cores was obtained from the voltage $V_{1}$ across an $1 \Omega$ resistance connected to the exciting coil in series. The induced voltage of the pickup coil was purified by a low pass filter with cutoff frequency of $30 \mathrm{~Hz}$, then amplified with gain of $60 \mathrm{~dB}$, and filtered again. After these signal processing, the induced voltage $V_{B}$ was integrated to obtain the magnetic flux inside the ring cores. A set of minor hysteresis loops were measured by increasing the amplitude of wave current gradually.

For the in situ magnetic measurements at $563 \mathrm{~K}$, the temperature of samples was first increased from 300 to $423 \mathrm{~K}$ without neutron radiation in the rate of $0.2 \mathrm{~K} / \mathrm{min}$. The reactor power was then increased from 0 to $50 \mathrm{MW}$ with a rate of $0.5-1.0 \mathrm{MW} / \mathrm{min}$ and the temperature was increased from 423 to $563 \mathrm{~K}$ in the rate of $14 \mathrm{~K} / \mathrm{min}$. The neutron flux was approximately 0.5 $\times 10^{13} \mathrm{~cm}^{-2} \mathrm{~s}^{-1}$ and the total neutron fluence was 5 $\times 10^{19} \mathrm{~cm}^{-2}$. In situ magnetic measurements were carried out every three or four days at the reaclor power of $50 \mathrm{MW}$. The temperature of samples was monitored with the thermocouples inserted into the samples. The temperature of A533B steel and iron metal samples was stabilized at $562 \pm 1.5 \mathrm{~K}$ and $563 \pm \mathrm{I} \mathrm{K}$, respectively, by using a heater and regulating the helium pressure in the capsule.

\section{EXPERIMENTAL RESULTS}

\section{A. Temperature dependence of minor-loop coefficients}

In the previous study, ${ }^{12}$ the relations of Eqs. (1)-(6) were examined experimentally at room temperature for plastically deformed A533B steel. Minor-loop coefficients, $W_{F} ", W_{R}{ }^{\prime \prime}$, $H_{c}{ }^{\prime \prime}, R_{c}{ }^{\prime \prime}, X_{T}{ }^{\prime \prime}$, and $\chi_{s}{ }^{\prime \prime}$ have a good relation with true stress which corresponds to the residual internal stress due to dislocations. In this study, the in situ measurements in the nuclear reactor has been carried out at a higher temperature of $563 \mathrm{~K}$. Therefore, we first checked the temperature dependence of minor-loop coefficients in our laboratory before in 


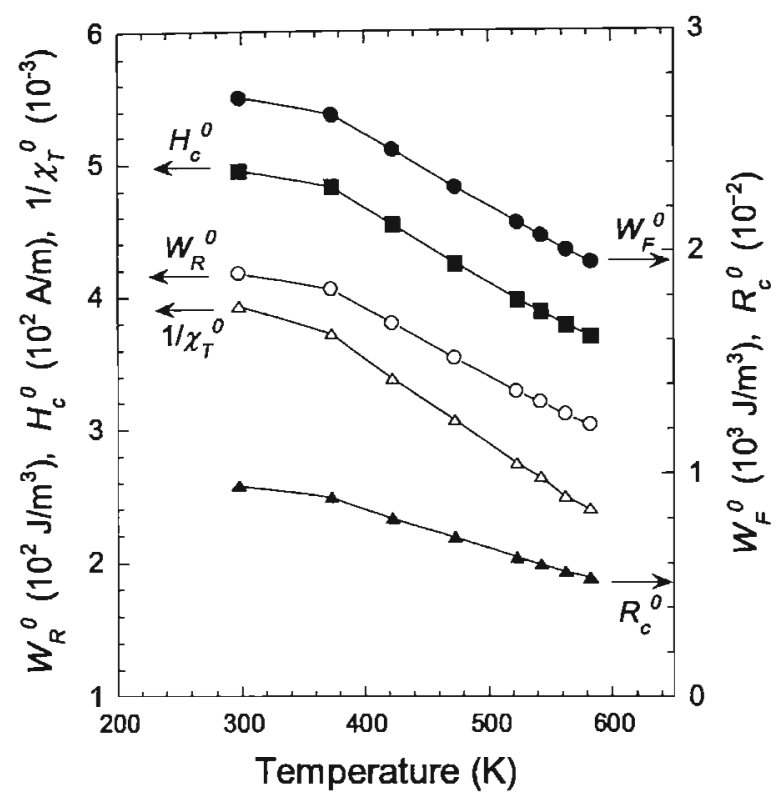

FIG. 4. Minor-loop coefficients, $W_{F}^{\prime \prime}, W_{R}{ }^{\prime \prime}, H_{c}{ }^{\prime \prime}, R_{r}{ }^{\circ}$, and $1 / \chi_{T}{ }^{\prime \prime}$, as a function of temperature in A533B steel.

situ measurements in the nuclear reactor. A set of minor loops was measured isothermally by using a flux meter in the temperature range from 300 to $583 \mathrm{~K}$. It was found that all minor-loop constants $n_{F}, n_{R}, n_{c}, b$, and $n_{T}$ are independent of temperature, and are $1.48,1.43,0.28,3.4 \pm 0.3$, and $0.13 \pm 0.01$, respectively. These values are consistent with those of plastically deformed A533B steels. As shown in Fig. 4. the minor-loop coefficients, $W_{F}{ }^{\circ}, W_{R}{ }^{0} . H_{c}{ }^{\circ}, R_{c}{ }^{\circ}$, and $1 / \chi_{T}{ }^{\circ}$ decrease monotonically with increasing temperature from 300 to $583 \mathrm{~K}$. The decreasing rate of $W_{R}{ }^{\circ}, W_{F}{ }^{\circ}$, and $H_{c}{ }^{\circ}$ at $563 \mathrm{~K}$ is about $0.15 \% / \mathrm{K}$, while that of $R_{c}{ }^{\circ}$ and $1 / \chi_{T}{ }^{\circ}$ is about $0.24 \% / \mathrm{K}$. These results were also obtained in the present in situ magnetic measurements in the nuclear reactor. Note that since $\chi_{a}{ }^{*}$ includes a large experimental error, the result of $\chi_{s}^{\circ}$ is omitted in this study.

\section{B. Minor-loop coefficients under neutron radiation}

The temperature of A533B steel sample was controlled automatically within $562 \pm 1.5 \mathrm{~K}$ throughout the radiation experiment. This yields a maximum deviation of $0.2 \%$ in $W_{R}{ }^{\prime \prime}$, $W_{F}^{\prime \prime}$, and $H_{c}^{\prime \prime}, 0.5 \%$ in $R_{c}^{\prime \prime}$, and $1 / \chi_{T}{ }^{\prime \prime}$ from the value at $T$ $=562 \mathrm{~K}$. Although this deviation did not change the fluence dependence of minor-loop coefficients significantly, the data were corrected for the temperature deviation to obtain an accurate value of the coefficients at $T=562 \mathrm{~K}$. It should be noted that temperature rise due to heat radiation of exciting coils during minor-loop measurements does not alfect experimental results. because the data below $0.8 \mathrm{kA} / \mathrm{m}$ were used for the minor-loop analysis and the rise of temperature was less than $0.5 \mathrm{~K}$.

Dependence of minor-loop coefficients on neutron fluence in Fe metal was obtained by analyzing a set of minor loops with Eqs. (1)-(6). Figure 5 shows minor-loop coefficients $W_{F}{ }^{\prime \prime}, W_{R}{ }^{\circ}$, and $H_{c^{\circ}}{ }^{\circ}$ as a function of neutron fluence in Fe metal. One can see that all coefficients increase monotonically with the increase of neutron fluencc. Their increase

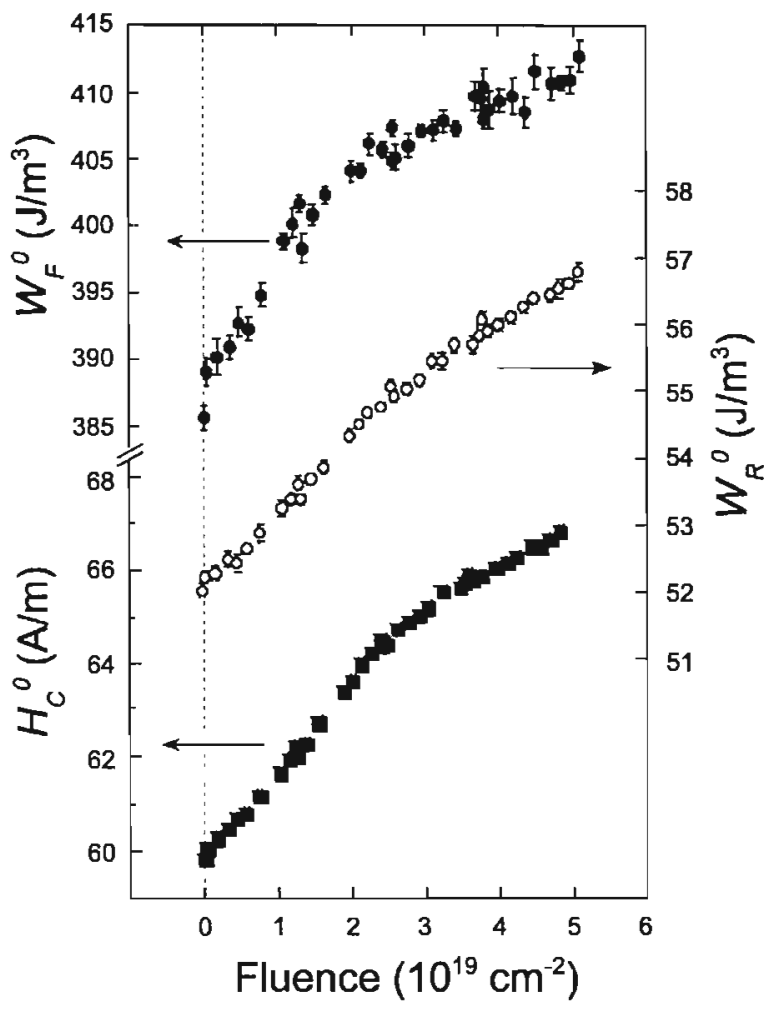

FIG. 5. Neutron fluence dependence of $W_{F}{ }^{\prime \prime}, W_{R}{ }^{\prime}$, and $H_{c}{ }^{\prime}$ in Fe metal.

corresponds to the increase of stress field and is consistent with the fact that the irradiation damage increases during neutron radiation. ${ }^{1}$

However, in A533B steel, the dependence of minor-loop coefficients on neutron fluence is largely different from that of Fe metal. As shown in Fig. 6, minor-loop coefficients $W_{F}{ }^{\circ}$,

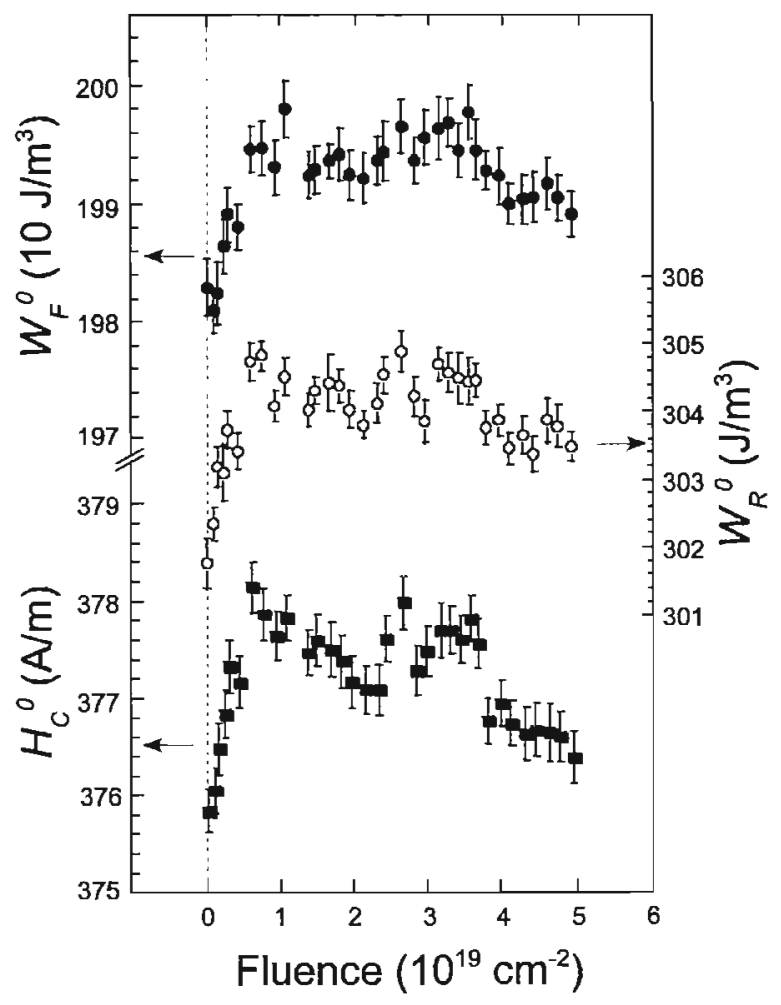

FIG. 6. Neutron fluence dependence of $W_{F}{ }^{\prime \prime} . W_{R}{ }^{\prime \prime}$. and $H_{c}^{\circ}$ in A533B steel. 


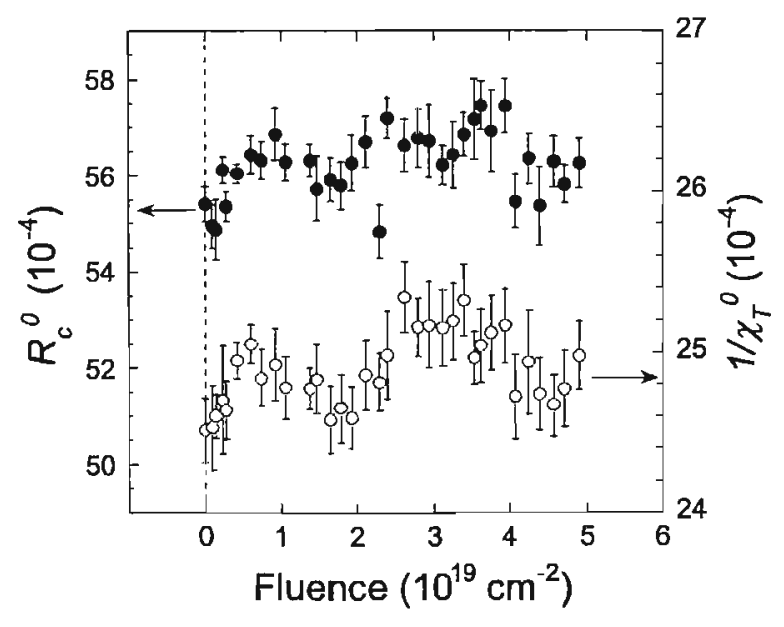

FIG. 7. Neutron fluence dependence of $R_{r}{ }^{\circ}$ and $1 / \chi_{T}{ }^{\circ}$ in A533B steel.

$W_{R}{ }^{\circ}$, and $H_{c}{ }^{\circ}$ sharply increase due to the radiation and show a maximum at the fluence of $1 \times 10^{19} \mathrm{~cm}^{-2}$. With increasing the neutron fluence, these coefficients slowly decrease and seem to have another local maximum around $3 \times 10^{19} \mathrm{~cm}^{-2}$. As shown in Fig. 7, such maxima were observed also for $1 / \chi_{T}{ }^{\circ}$. although the maximum around $3 \times 10^{19} \mathrm{~cm}^{-2}$ was not clearly seen for $R_{c}$. . The maximum of minor-loop coefficients at the initial stage of neutron irradiation has been observed also in Fe model alloys after neutron irradiation. ${ }^{14}$ The decrease of the minor-loop coefficients indicates that the irradiation damage decreases above $1 \times 10^{19} \mathrm{~cm}^{-2}$, contrary to the results of Fe metal.

\section{DISCUSSION}

The minor-loop coelficients are a function of modulus of rigidity, Poisson ratio, magnetostriction constant, and magnetic anisotropy constant. and their temperature dependence gives us information on their functional structure. As shown in Fig. 4, all coefficients decrease linearly with temperature and show a similar temperature dependence. Therefore, the minor-loop coefficients would have a similar functional structure of such physical properties.

Minor-loop coefficients $W_{F}{ }^{\circ}, W_{R}{ }^{\prime \prime}$, and $H_{c}{ }^{\prime \prime}$ are sensitive to the size and shape of Bloch wall potential, while those related to susceptibilities. $R_{c}{ }^{\circ}, 1 / \chi_{T}{ }^{\circ}$ and $1 / \chi_{s}{ }^{\circ}$, depend on the resistance against the movement of $B$ loch wall at the special point of potential. Generally, the coefficients are sensitive to dislocations because of magnetoelastic interaction between domain walls and dislocations. ${ }^{8}$ The minor-loop coefficients also give information about irradiation damage due to other lattice defects such as copper precipitates and dislocation loops, because these defects also yield stress field around them.

In Fe metal, all minor-loop coel'ficients $W_{F}^{\prime \prime}, W_{R}^{\prime \prime}$, and $H_{c}{ }^{\prime}$ increase monotonically during neutron radiation as shown in Fig. 5. The irradiation damage in Fe metal would be due 10 dislocation loops, vacancies, and interstitial atoms. Since the size of vacancies and interstitial atoms are too small to exert an influence to Bloch wall motion, only dislocation loops would play an important role for obstacles to the Bloch wall motion in Fe metal. The number and size of dislocation loops increase with the increase of neutron fluence, resulting in the monotonic increase in the minor-loop coefficients. On the other hand, in A533B steel, minor-loop coefficients increase during neutron radiation and show a maximum at the fluence of $1 \times 10^{19} \mathrm{~cm}^{-2}$, followed by a slow decrease with neutron fluence. This change in the minor-loop coefficients indicates that the residual internal stress decreases and obstacles to the Bloch wall motion become small above the fluence of $1 \times 10^{19} \mathrm{~cm}^{-2}$.

The metallurgical differences between A533B steel and Fe metal are chemical compositions and the value of $\rho$; the value of $\rho$ for A533B steel is above $3 \times 10^{10} \mathrm{~cm}^{-2}$ and is much higher than the value of Fe metal. ${ }^{15}$ The radiation defects that yield the brittleness in A533B steel would be copper precipitates and dislocation loops, since they act as obstacle to the dislocation movement. Copper precipitates and dislocation loops do not diminish with the increase of neutron fluence eventhough the density of such defects is saturated at a high fluence.

Generally, mechanical properties such as yield stress, Vickers hardness, and ductile-brittle transition temperature (DBTT) increase with neutron fluence for all materials. It was confirmed for present A533B steel that both Vickers hardness and DBTT increase after neutron irradiation with the fluence of $5 \times 10^{19} \mathrm{~cm}^{-2}$. Such a change of mechanical properties can be explained by the dislocation theory and is contradictory to the minor-loop properties in A533B steel.

The maximum of minor-loop coefficients in A533B steel would be explained by the introduction of an idea that copper precipitates grow in the vicinity of dislocations by neutron irradiation. Copper precipitates and dislocation loops in the matrix have stress field in themselves and make the minor-loop coefficients increase. On the other hand, the stress field is reduced and minor-loop coefficients decrease when these two lattice defects exist in the vicinity of dislocations. Edge dislocations include the compressive and repulsive stress field, and both dislocation loops and copper precipitates may gather around the dislocations through the elastic interaction. Both radiation defects will compensate the stress ficld of dislocations and this compensation brings about a reduction in the elastic energy. The emulation of these two effects of stress field in the matrix and around dislocations would result in the maximum of minor-loop coefficients. These radiation defects fixed around dislocations strongly disturb the dislocation movement and make mechanical properties increase as was observed experimentally.

In the same way. dislocation loops nucleate and grow during neutron radiation, also in Fo metal. However. dislocation density before neutron radiation, estimated from coercive force of $60 \mathrm{~A} / \mathrm{m}$. is $7 \times 10^{8} \mathrm{~cm}^{-2}$ and is very small compared with that of $\mathrm{A} 533 \mathrm{~B}$ stcel. Therefore, the stress field of dislocations may be compensated partially by dislocation loops and the net stress field increases during neutron radiation.

Quite recently, small dislocation loops and copper precipitates in the vicinity of dislocations have been observed 
by TEM in A533B steel after neutron irradiation with the fluence of $5 \times 10^{19} \mathrm{~cm}^{-2}$. The density of dislocation loops and copper precipitates in the vicinity of the dislocations was found to be higher than that in the matrix. The results by TEM observation will appear in a separate paper.

\section{CONCLUSION}

In situ magnetic measurements have been successfully carried out under neutron radiation at $563 \mathrm{~K}$. We found that the nucleation mechanism of radiation defects in $\mathrm{Fe}$ metal and $\mathrm{A} 533 \mathrm{~B}$ steel is largely different from each other.

In Fe metal, minor-loop coefficients increase monotonically with the increase of neutron fluence. Dislocation loops which nucleate and grow during neutron radiation, make the minor-loop coefficients increase through magnetoelastic coupling.

On the other hand. in A533B steel, the minor-loop coefficients increase initially and show a maximum at the neutron fluence of $1 \times 10^{19} \mathrm{~cm}^{-2}$, followed by a slow decrease with neutron fluence. This indicates the presence of two mechanisms of internal stress due to radiation defects in $\mathrm{A} 533 \mathrm{~B}$ steel. The copper precipitates and dislocation loops gathering in the vicinity of dislocations would disturb the dislocation movement and make A533B steel brittle.

\section{ACKNOWLEDGMENTS}

The authors express thanks to Professor G. R. Odette of University of California, Santa Barbara for valuable discussion on the interaction between dislocations and lattice defects. This research was supported by a Grant-in-Aid for Scientific Research (S), Grant No. 141020345, from the Ministry of Education, Culture, Sports. Science and Technology of Japan.

'J. Koutský and J. Koik, Radiation Damage of Structural Materials (Elsevier Science, New York, 1994).

'P. Othen, M. Jenkins, G. Smith, and W. Phyltian, Philos. Mag. Lett. 64, 383 (1991).

${ }^{3}$ S. Pizzini. K. Roberts. W. Phythian. C. English. and G. Greaves. Philos. Mag. Lell. 61. 223 (1990).

${ }^{4}$ A. Youle and B. Ralph, Met. Sci. J. 6, 149 (1972).

s. G. Goodman, S. Brenner, and J. R. Low. Jr., Metall. Trans. 4. 237I (1973).

${ }^{6}$ I. Altpeter. G. Dobmann, K.-H. Katerbau. M. Schick, P. Binkele, P. Kizler, and S. Schmauder, Nucl. Eng. Des. 206, 337 (2004).

${ }^{7}$ M. K. Miller. B. D. Wirth. and G. R. Odette, Mater. Sci. Eng., A 353. 133 (2003).

${ }^{8} \mathrm{H}$. Kronmüller and M. Fähnle. Micromagnetism and the Microstrucure of Ferromagnetic Solids (Cambridge University Press, Cambridge, 2003).

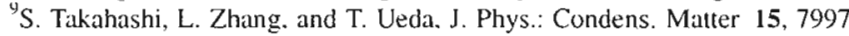
(2003).

${ }^{10}$ S. Takahashi, T. Ueda, and L. Zhang, J. Phys. Soc. Jpn. 73, 239 (2004).

${ }^{11}$ S. Takahashi and L. Zhang, J. Phys. Soc. Jpn. 73. 1567 (2004).

${ }^{12}$ S. Takahashi. L. Zhang. S. Kobayashi. Y. Kamada, H. Kikuchi, and K. Ara. J. Appl. Phys. 98. 033909 (2005).

${ }^{13}$ C. P. Steinmetz, Theory and Calculation of Electric Circuits (McGrawHill. New York, 1917).

${ }^{14}$ S. Kobayashi, H. Kikuchi, S. Takahashi. K. Ara, Y, Kamada (unpublished).

${ }^{15}$ S. Takahashi. J. Echigoya. and Z. Motoki. J. Appl. Phys. 87, 805 (2000). 